\title{
Neue Wege in der dermatologischen Weiterbildung \\ Teil 1: Wein und Haut - Degustation für die Ärzte der Hautklinik im BASF-Weinkeller
}

New Paths in Dermatologic Education

Part 1: Wine and Skin - Winetasting for Dermatologists in the BASF-Wine Cellar

Autoren

Institute

\section{Löser ${ }^{1}$, J. Wolf ${ }^{2}$}

Hautklinik, Hauttumorzentrum, Klinikum Ludwigshafen

2 Neurologische Klinik, Klinikum Ludwigshafen

\section{Bibliografie}

DOI http://dx.doi.org/

10.1055/s-0030-1256635

Online-Publikation: 28. 7. 2011

Akt Dermatol 2011; 37:

306-308 ๑ Georg Thieme

Verlag KG Stuttgart · New York ISSN 0340-2541

\section{Korrespondenzadresse} Dr. med. Christoph Löser Leitender Oberarzt der Hautklinik/Hauttumorzentrum Rheinpfalz

Klinikum Ludwigshafen

Bremserstraße 79

67063 Ludwigshafen

loeserc@klilu.de

\section{Zusammenfassung \\ $\nabla$}

Die Dermatologie stellt an den Facharzt den hohen Anspruch, Strukturen und Zusammenhänge wahrzunehmen, die auf der Haut jeder sehen kann, aber nur das geschulte Auge erkennt. Vor diesem Hintergrund wurden an der Hautklinik Ludwigshafen im Jahr 2010 neben den zahlreichen fachbezogenen Fortbildungen auch zwei Veranstaltungen durchgeführt, welche die Sensorik auf unkonventionelle Weise fördern sollten.

Wie im Wein die Düfte und Noten

sind auf der Haut die Blüten uns Boten.

Christoph Löser

\section{Alkohol und Haut \\ $\nabla$}

Alkohol ist im christlichen Abendland eine legale, billige Droge, die sozial so akzeptiert ist, dass sich - anders als beim Rauchen - der entschuldigen muss, der nicht trinkt. Vielfältige gesundheitliche Risiken sind wohlbekannt und reichen von Abhängigkeit und Alkoholismus über Organschädigungen bis hin zum Tod bei alkoholisierter Teilnahme am Straßenverkehr. Der Androloge kennt die Steigerung der Libido beim Mann, die allerdings nicht mit der Standfestigkeit korreliert, da ab 0,4 Promille Erektionsstörungen auftreten. Darüber hinaus kommt es zu Fertilitätsstörungen, wenn der Spiegel an Östrogen steigt und Testosteron sinkt. An der Haut führen direkte Wirkungen zum Auftreten eines Flushs, der als Rubeosis faciei chronifizieren kann und als Stigma fungiert (Facies alcoholica). Zu den direkten Wirkungen auf die Haut zählt auch eine Seborrhoe und aufgrund der entwässernden Wirkung die Exsikkation. Klassische Hautmanifestationen alkoholtoxischer Effekte sind Palmarerythem, Spider naevi, Fettverteilungsstörungen, Haarverlust, Elastolyse,
Im ersten Halbjahr fand im Gesellschaftshaus der BASF zunächst eine Weinprobe statt, die von Fachvorträgen zu den Themen Alkohol und Haut sowie Wein und Gesundheit begleitet wurden. Unter der fachkundigen Anleitung von Dr. Joachim Wolf, geschäftsführender Oberarzt der neurologischen Klinik, wurde der Geschmackssinn geschärft und die Analogie von Schmecken und Sehen auf genussvolle wiewohl maßvolle Weise untersucht.

Hämorrhagie, Atrophie (paper money skin) und Pruritus. Ein übermäßiger Alkoholkonsum begünstigt verschiedene Dermatosen, dazu zählen Psoriasis, Seborrhoisches Ekzem, Nummuläres Ekzem und die Rosazea. Als Folge des Alkoholismus kann es weiterhin zum Auftreten von Porphyria cutanea tarda, Acroosteopathia ulceromutilans (Bureau-Barrière), Infektionen (viral, mykotisch, bakteriell), Xerosis, Impetigo, Follikulitiden und eruptiven Xanthomen kommen.

Polyphenolen im Wein werden photoprotektive Effekte zugeschrieben, und tatsächlich konnte bei einem Pilotversuch in Tübingen die Steigerung der Erythemschwelle durch Rotweinkonsum gezeigt werden, während topische Effekte nicht nachgewiesen wurden [1].

\section{Alkohol und Gesundheit}

$\checkmark$

Ein moderater Alkoholkonsum hingegen hat durchaus auch seine positiven Seiten. Das Sterblichkeitsrisiko von Menschen, die ungefähr 10 12 Gramm Alkohol ( $=1$ „Drink“ $\approx 0,11$ Wein) täglich konsumieren, liegt unter dem abstinent lebender Menschen, aber auch unter dem Risiko derjenigen, die deutlich höhere Alkoholmengen zu sich nehmen [2]. Besonders deutlich wird dies bei kardiovaskulären Erkrankungen. So haben zahlreiche wissenschaftlich hohen Ansprüchen 
genügende Studien gezeigt, dass moderater Alkoholgenuss das relative Herzinfarktrisiko drastisch senkt [3]. Ähnliches gilt auch für das Risiko, an einem Diabetes mellitus Typ II oder an einer vaskulären oder Alzheimer-Demenz zu erkranken [4]. In Bezug auf Schlaganfälle ist Alkoholkonsum ein zweischneidiges Schwert. So schützt moderater Alkoholgenuss zwar vor Hirninfarkten, erhöht jedoch das Risiko, eine Hirnblutung zu erleiden [5].

Wie erklärt sich nun der günstige Effekt des Alkohols bei Gefäßerkrankungen? Einerseits wirkt er positiv auf den Cholesterinstoffwechsel, indem er HDL-Cholesterin erhöht. Andererseits hemmt er ähnlich der Acetylsalicylsäure die Thrombozytenaggregation und senkt Fibrinogen. Zusätzlich wirkt Alkohol antientzündlich.

Die Kehrseite der Medaille soll jedoch nicht unerwähnt bleiben. Bereits in moderaten Mengen kann Alkohol das Risiko, an bestimmten Krebsarten zu erkranken, erhöhen! Dies gilt insbesondere für das Brustkrebsrisiko [6]. Bereits moderate Alkoholmengen erhöhen das relative Risiko um $10 \%$.

Was verstehen Experten unter „moderaten“ Alkoholmengen? Die Deutsche Gesellschaft für Ernährung (DGE) empfiehlt eine maximale Menge von $10 \mathrm{~g}$ Alkohol/d für Frauen und $20 \mathrm{~g}$ Alkohol/d für Männer, während die Deutsche Weinakademie für Frauen maximal $20 \mathrm{~g}$ Alkohol/d und für Männer höchstens $30 \mathrm{~g}$ Alkohol/d vorschlägt. Letztlich sollte die täglich konsumierte Menge nicht über einem viertel Liter Wein liegen und dies am günstigsten zu den Mahlzeiten.

Das Leben ist zu kurz,

um schlechten Wein zu trinken.

Johann Wolfgang von Goethe

\section{Weininhaltsstoffe}

Die 1991 in den USA ausgestrahlte Fernsehsendung „Sixty Minutes“ zum Thema „Wein und Gesundheit“ mit Prof. Renaud aus Lyon ließ den Weinabsatz in Nordamerika in der Folge sprunghaft um $40 \%$ ansteigen. In dieser Sendung wurden die Hintergründe des Begriffs „French paradox“ erstmals einer breiten Öffentlichkeit dargestellt. Prof. Renaud zitierte damals Ergebnisse aus Studien, wonach die kardiovaskuläre Sterblichkeit in Frankreich deutlich niedriger sei als in anderen westlichen Ländern, obwohl Franzosen mehr tierische Fette verzehrten, stärker rauchten und weniger Sport trieben. Als Grund führte er den höheren Weinkonsum unserer französischen Nachbarn an. In der Tat scheint Wein bei gleicher Alkoholmenge gesundheitlich günstigere Eigenschaften aufzuweisen als Bier oder Spirituosen, wie die Kopenhagen-City-Studie zeigte [7]. Es ist natürlich nicht ausgeschlossen, dass Menschen, die Wein präferieren, auch sonst gesünder leben als Bier- und Spirituosentrinker. Möglicherweise spielen jedoch auch noch andere Weininhaltsstoffe neben dem Alkohol eine wichtige Rolle. Hier rücken zunehmend Polyphenole in den Mittelpunkt, insbesondere Quercetin, Procyanidin und trans-Resveratrol, die in vitro und in Tierversuchen wie Alkohol selbst günstige Eigenschaften auf das kardiovaskuläre System aufweisen. Resveratrol und Quercetin haben zusätzlich auch antimutagene und antikarzinogene Wirkungen [8], und Resveratrol wird in Tierversuchen sogar eine lebensverlängernde Wirkung zugeschrieben [9]. Die Konzentration der Polyphenole im Wein ist zunächst abhängig von der Traubensorte, jedoch

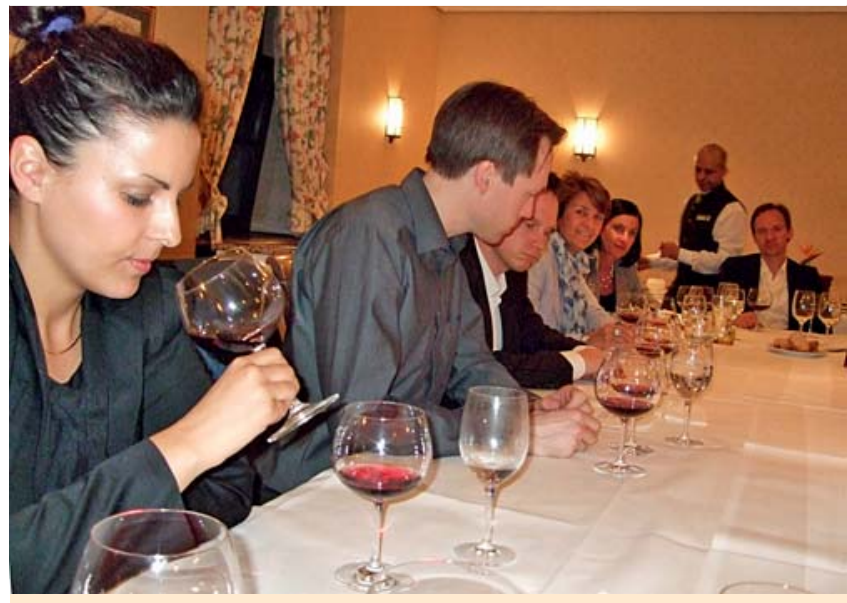

Abb. 1 Rotwein-Probe mit Ärzten der Hautklinik Ludwigshafen.

auch von der Art des Weinausbaus (verlängerte Maischestandzeit und Maischegärung bei Rotweinen). Ob allerdings die geringen Konzentrationen der Polyphenole im Wein ausreichen, um gesundheitsfördernde Effekte zu erreichen, ist zweifelhaft.

Letztendlich stellte bereits der Arzt Paracelsus von Hohenheim (1493-1541) treffenderweise fest: „Alle Dinge sind Gift, und nichts ist ohne Gift. Allein die Dosis macht, dass ein Ding kein Gift ist." Dem ist beim Weingenuss nichts hinzuzufügen.

Die Wirkungen des Weingenusses auf den menschlichen Körper und Geist lassen sich am besten und genussreichsten bei einer kleinen Weinprobe erleben, wie sie im April 2010 im Gesellschaftshaus der BASF in Ludwigshafen stattfand. Sie sollte auch dazu dienen, die sensorischen Fähigkeiten der Teilnehmer zu schärfen.

\section{Weinsensorik (Weinbewertung mit Hilfe der Sinne) $\nabla$}

Das Verkosten eines Weins stützt sich im Wesentlichen auf die menschlichen Sinnesleistungen Sehen, Riechen und Schmecken. Mit den Augen lassen sich wichtige Eigenschaften des Weins wie Farbe und Klarheit erfassen. Der Farbton und die Intensität der Farbe können etwas über das Alter des Weins verraten. Gekonntes Schwenken des Weins im Glas führt zu einer Benetzung und zu einem anschließenden Herabrinnen an der Glasinnenfläche. Weine hinterlassen je nach Viskosität sehr unterschiedliche Rinnspuren. Die Viskosität eines Weins ist ein Indikator für seinen Gehalt an Alkohol und Extrakt und damit ein Qualitätskriterium. Das Schwenken des Weinglases dient auch der Verflüchtigung von Weinaromastoffen, die so besser von der Nase erfasst werden können. Das menschliche Riechorgan ist in der Lage, ca. 350 verschiedene Aromen zu unterscheiden, eine im Vergleich zur Tierwelt recht geringe Anzahl. Die Aromen des Weins sind von vielen Faktoren abhängig, natürlich von der Traubensorte, aber auch von der Art des Weinausbaus im Weinkeller und vom Alter des Weins.

In der Weinprobe ( $\bullet$ Abb. 1) konnten wir Aromen von Früchten und Gemüse erschnuppern.

So fanden sich Düfte von Pfirsich und Grapefruit beim Riesling, Noten von Stachelbeere, Spargel und Paprika beim Sauvignon blanc, solche von Erdbeeren und Brombeeren beim Spätburgunder und Aromen schwarzer Früchte und Paprika beim verkosteten Syrah. Bei einigen Weinen waren zusätzlich die Gesteinsformation des Weinbergs und die Art des Weinanbaus olfaktorisch 


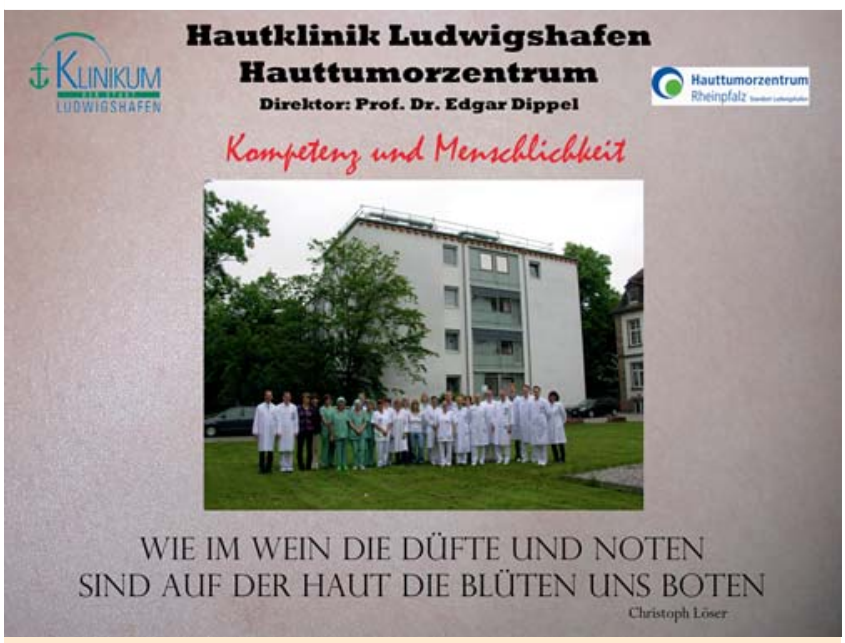

Abb. 2 Wein-Etikett der Hautklinik Ludwigshafen.

erfassbar. Wir erkannten ein Aroma von Feuerstein beim Riesling aus der mit vulkanischem Basalt durchsetzten Forster Lage Pechstein, Leder-Noten beim Pinot Noir von der burgundischen Côte de Beaune und Gewürz-Noten und Tabakaromen beim Syrah von der Rhone.

Der erste Schluck Wein führt zu einem komplexen Geschmackseindruck, obwohl der Mensch nur wenige Geschmacksempfindungen wahrnehmen kann. So sind Süße und Säure des Weines über die Zunge erfassbar. Salzige und bittere Noten spielen eine untergeordnete Rolle. Hingegen wirkt ein gerbstoffbetonter Wein an der gesamten Mundschleimhaut adstringierend. Gewöhnungsbedürftiges Schlürfen des Weins im Mund ist durchaus erlaubt, da so mehr flüchtige Aromastoffe freigesetzt und über den Nasen-Rachen-Raum zur Riechschleimhaut transportiert werden (retronasale Atmung), was den Geruchseindruck verstärkt. Nach dem ersten Schluck bleibt je nach Güte des Weins ein mehr oder weniger langer Nachgeschmack im Mund zurück (Abgang), was wir besonders beim edelsüßen Pfälzer Riesling aus Forst erfahren durften.

Nach der zehnteiligen Weinprobe, die von kulinarischen Genüssen begleitet wurde, war zumindest unser sensorischer Geist auf Hochtouren.

\section{Fazit}

$\nabla$

Alkohol und insbesondere Wein in moderaten Mengen besitzen auch positive Effekte. Allerdings dient der Wein dem sinnlichen Genuss und ist weder Arzneimittel noch Nahrungsmittel im eigentlichen Sinne. Dennoch wird mancher in einer Risiko-Nutzen-Abwägung ähnlich wie beim Autofahren trotz aller Gefahren auf die positiven Wirkungen nicht verzichten wollen. In der Dermatologie lässt sich ein moderater Weingenuss vielleicht sogar mit der Schulung der Sensorik als Training von Wahrnehmung entschuldigen.

Inzwischen besitzt die Hautklinik eine eigene Kollektion von Weinetiketten, mit denen ausgewählte Tropfen versehen und an besondere Gäste oder eingeladene Redner überreicht werden können ( $\bullet$ abb. 2). Gerüchte, dass der Wein von einem Klinikeigenem Weinberg stammt, welcher den Mitarbeitern der Hautklinik Ludwigshafen aufgrund guter Zahlen geschenkt wurde und den sie nach Feierabend zur Entspannung selbst bestellen, müssen allerdings in das Reich der Sagen und Fabeln verwiesen werden.

\section{Abstract}

\section{New Paths in Dermatologic Education Part 1: Wine and Skin - Winetasting for Dermatologists in the BASF-Wine Cellar $\nabla$}

Dermatology demands from its proponents to recognize structures and connections, everyone can see on the skin, but only the trained eye will recognize. Therefore in 2010 the physicians of the Skin Hospital in Ludwigshafen were invited in addition to several conventional educational meetings to train their senses in a most exceptional way.

Here they went to a wine-tasting, accompanied by lectures regarding wine and health as well as skin and alcohol. Under the tutelage by Dr. Joachim Wolf, they honed their senses and the analogy between taste and look was explored in a pleasurable albeit modest way.

\section{Literatur}

1 Möhrle M, Dietrich H, Patz CD, Häfner HM. Lichtschutz durch Rotwein? J Dtsch Dermatol Ges 2009; 7: 29-33

2 Di Castelnuovo A, Costanzo S, Bagnardi V et al. Alcohol dosing and total mortality in men and women: an updated meta-analysis of 34 prospective studies. Arch Intern Med 2006; 166 (22): 2437-2445

3 Corrao G, Rubbiati L, Bagnardi Vet al. Alcohol and coronary heart disease: a meta-analysis. Addiction 2000; 95: 1505 - 1523

4 Mukamal KJ, Kuller LH, Fitzpatrick AL et al. Prospective study of alcohol consumption and risk of dementia in older adults. JAMA 2003; 289: $1405-1413$

5 Elkind MS, Sciacca R, Boden-Albala B et al. Moderate alcohol consumption reduces risk of ischemic stroke: the Northern Manhattan Study. Stroke 2006; 37 (1): 13-19

6 Smith-Warner SA, Spiegelman D, Yaun SS et al. Alcohol and breast cancer in women: a pooled analysis of cohort studies. JAMA 1998; 279 (7): $535-40$

7 Gronbaek M, Becker U, Johansen D et al. Type of alcohol consumed and mortalitiy from all causes, coronary heart disease and cancer. Ann Intern Med 2000; 133: 411-419

8 Baur JA, Sinclair DA. Therapeutic potential of resveratrol: the in vivo evidence. Nat Rev Drug Discov 2006; 5: 493 - 506

9 Howitz KT, Bitterman KJ, Cohen HY et al. Small molecule activators of sirtuins extend Saccharomyces cerevisiae lifespan. Nature 2003; 425: $191-196$ 